\title{
Anxiety and depression disorders in cancer patients: incidence, diagnosis and therapy
}

\section{Fischer, B. Wedel}

Universitätsklinikum Schleswig-Holstein, Campus Lübeck, Ratzeburger Allee, Lübeck, Germany

Received 2 November 2011; accepted 16 January 2012

Psycho-oncologist research in recent years has focused on assessment of distress and mental disorders in cancer patients. Primary objective has been the implementation of individually tailored psychosocial support during routine medical care. This review shows that based on validly structured clinical interviews, $30-40 \%$ of the studied patient population had a mental disorder in accordance with the updated Diagnostic and Statistical Manual of Mental Disorders (DSM-IV). Most common diagnoses were depression, adjustment and anxiety disorders. Risk factors included among others younger age, previous history of mental disorders and lack of social support. Screening instruments are useful, easy to complete and allow early detection of high distress in patients, enabling appropriate interventions in affected patients. Established methods in psycho-oncology are cognitive behavioural therapy, psycho-educational groups and relaxation trainings. A positive influence of early palliative care intervention on cancer patients' mood has been shown. Adequate resources in acute and outpatient routine care shall be offered.

Keywords: Distress, psycho-oncology, quality of life, supportive care, underestimation.

\section{Introduction}

Few diseases are as strongly affiliated with fear as cancer. The emotional reactions range from moderate worry to disorders that meet the full DSM-IV criteria [1]. They are commonly caused by the severity of physical complaints and not related to neurotic conflicts. Although psychosocial care is becoming more widely available due to higher numbers of specialized cancer centres, the extent of distress is often not identified and therefore not adequately treated [2]. This can lead to increased mortality [3], reduced quality of life [4], worsening of compliance [5] and prolonged hospitalization [6]. Underestimation of emotional side effects is caused by deficient training in recognizing distress [7] and an inability to communicate

Correspondence: Dorothea Fischer, MD, Universitätsklinikum Schleswig-Holstein, Campus Lübeck, Ratzeburger Allee 160, 23562 Lübeck, Germany.

E-mail: dorothea.fischer@uksh.de emotional issues [8], a lack of time for sufficient contact as well as economical and institutional circumstances [9].

Patients expect the physician to address such topics actively since the majority fears additional stigmatization through a psychiatric diagnosis when they communicate such problems themselves. Complicating the matter further, the interest of cancer patients in psychological support correlates more with gender, age and individual coping strategies rather than a high burden of distress or the presence of a psychiatric diagnosis [10].

Therefore comprehensive care for cancer patients requires knowledge about psychiatric disorders as well as detailed diagnostic and therapeutic skills to provide a competent treatment of both somatic and psychological needs.

\section{Epidemiology}

To develop cancer exceeds coping resources in $30-40 \%$ of the affected persons [1], leading to one $(20 \%)$ or more (11.6\%) mental disorders [11]. The most frequent diagnoses involve affective disorders: major or minor depression or dysthymia. A recently published meta-analysis (94 interview-based studies with 14,078 individuals in hospital settings) revealed a lesser prevalence when diagnosis was achieved in interviews as opposed to other methods such as screening questionnaires [1]. Point prevalence of affective disorders in general population is $6 \%$ in men and $10 \%$ in women. The percentages in cancer patients are much higher with $20.7 \%$ and $24.6 \%$ in palliative care settings, respectively [1]. Other authors describe even 3-5 times higher incidence than in the general population [12]. Interestingly, anxiety disorders by DSM or ICD criteria (like generalized anxiety disorder, panic disorder, agoraphobia, specific or social phobia) do not occur more frequently in cancer patients (2-16\%) [11] than in the general population (5\% in men, $12 \%$ woman) [13] during acute care. Adjustment disorders are found notably more frequently in cancer patients (15-19\%) [1], point prevalence of posttraumatic disorder occurs in 3-6\% [14]. A current meta-analysis [1] found no effect of age, sex or clinical setting.

\section{Risk factors}

The development of psychiatric co-morbidity in cancer patients is best described as a continuum beginning with small 
distress, followed by adjustment disorder and finally leading to depressive or anxiety disorder. Risk factors are categorized into biological, psychological and social components. For example, the individual aetiology of the often noticeable fatigue syndrome, which appears similar to depressive mood, is possibly caused by neurobiological or hormonal changes as well as by tumour or therapy induced influences [16]. Important to consider are the depressive symptoms as unwanted side effects after medical treatment, e.g. with cytokine IL-2 or interferonalpha (IFN- $\alpha$ ), observed in one-third of patients under therapy [17]. Psychological factors include among others coping strategies or previous history of mental disorders. Social factors like familiar or social support play an important role, too.

Prognostic relevant risk factors for depression are biological factors like younger age, previous history of depression and advanced disease as well as psychological factors like subjective perceived social support, less optimism, less self confidence and social factors like a lack of social support, recent losses and previous history of trauma [18].

Risk factors for adjustment disorders are advanced and severe disease, worse prognosis, early onset, low functional status, pain, living alone and worry about being strain for others [15] as well as having underage children [19].

\section{Diagnostic of mental disorders}

It has been shown that most patients do not express their emotional concerns and needs for psychosocial support [10]. Reasons for that are often fear of additional stigmatization or denial. To disregard anxiety is not as feasible as disregarding depression, which could be interpreted as adjustment to disorder. Interaction effects between disease- or therapy-induced symptoms and mood variations possibly complicate assessment of mental disorders, often observed during shock of primary diagnosis, ongoing chemotherapy or under vigorous pain [19]. This leads to under- or overestimation of prevalence resulting in a significant lack of support [20].

Cancer-specific screening instruments were developed to detect patients with significant needs. They shall be used ahead of or at least parallel to existing somatic diagnostic routine and shall be applied to each patient systematically according to international guidelines [21].

Established instruments are the Hospital Anxiety and Depression Scale, HADS [22] and the NCCN-distress thermometer. The most common valid, but time-consuming instrument is the "Structured Clinical Interview for DSM-Disorders, SCID” [23].

\section{Acute stress disorder}

The symptoms of crisis (psychological shock, emotional numbing, restriction of consciousness, inability to handle stimuli, vegetative arousal) start within minutes and ease within hours or days. Most patients cope with this situation by themselves. Assistance from personnel who are also competent with respect to medical issues is very helpful in that situation.

\section{Adjustment disorders}

Adjustment disorders with both depressive or anxiety symptoms arise when adaptation was not successful and should only be diagnosed when no other major mental disorder or bereavement exists. The perception of not coming back to routine after the distressing event and significant impairment in functioning is central. Symptoms of irritability, aggressiveness and sleep disorders can strongly affect the cancer patients. The disorder lasts generally for several months and shall be treated by psychotherapeutic interventions.

\section{Posttraumatic stress disorder (PTSD)}

PTSD is a reaction to a traumatic event of a life-threatening illness such as cancer. Those patients often present very frightened, helpless or horrified state. This category may only be assigned within six months from the appearance of the event and in absence of anxiety or depressive disorders. Three leading symptoms are characteristic: intrusion, avoidance and arousal. Effective therapeutic approaches are, for example, EMDR (Eye Movement Desensitization and Reprocessing) and imaginative techniques to stabilize the patients.

\section{Anxiety}

Anxiety has cognitive appraisal, affective, behavioural and physiological components, which are sometimes difficult to distinguish from specific side effects of therapy, e.g. nausea.

In the context of cancer, anxiety is adaptive and activates resources to avoid danger.

Maladaptive anxiety leads to inappropriate thoughts, activities or impairments. Debilitating fear of recurrence, exacerbation of previous anxiety disorder or therapy-induced traumata in acute care like lack of oxygen in lung cancer can lead to maladaptive anxiety. Anxiety can be alleviated through psycho-educative interventions, e.g. "normalizing" of symptoms or clarification of the immediate contents. In acute hospital care it is common to use anxiolytics, which should not be used in outpatient, non-palliative settings because of their highly addictive potential.

\section{Depression}

Sadness after confrontation with cancer disease or recurrence is adequate. However, clinically significant depression with leading symptoms of depressed mood, loss of interest and anhedonia is often not sufficiently recognized and treated. Depression causes decreased quality of life and affects compliance which possibly leads to adverse process of disease [25] and prolonged hospitalization [6]. Diagnostic distinguishes between depressive reaction within adjustment disorders, major or minor depressive episode, dysthymia and cancer-specific impairments of mood. Half of affected patients remain undetected [26] although appropriate treatment is needed and effective. Patients with a reliable diagnosis of major depression should be treated with combined therapy consisting of psychotherapy and psychopharmacotherapy. Important for choice of medication is age, general status, risk of interaction and profile of side effects. New antidepressants with more favourable profile of side effects, notably less risk of cytochrom p450-interaction, are used [27]. In case of agitate behaviours anxiolytics may be necessary; in case of delusion neuroleptics shall be used. However, at present the use of pharmacotherapy is not well examined in cancer care and there are no existing guidelines for differentiated prescrip- 
tions [28]. Although pharmacotherapy is first choice for physicians, patients mostly require psychotherapy [29].

\section{Differentiated diagnosis}

Before a mental diagnosis can be established, it is important to exclude somatic aspects like thyroid dysfunction, cerebral or neurological alterations. In addition it is necessary to consider sequelae of cancer disease (e.g. cachexia, anaemia) together with their impacts fatigue, decrease of concentration and reduced functionality. Recently two studies impressively could show the positive influence of early palliative care intervention on cancer patients' mood $[30,31]$. Therefore an early involvement of palliative care specialists would be reasonable.

\section{Therapy}

The goal of psycho-oncology is to reduce distress, anxiety and depression and to strengthen communication with the social environment, to rediscover resources and thereby enhance quality of life of cancer patients. Interventions are individually tailored to kind and severity of symptoms. In addition to psychotherapeutic interventions pharmaceutical therapies shall be used in major disorders. Results of different meta-analyses lead to evidence-grade-I for psycho-oncological interventions [32]. Cognitive-behavioural and supportive-expressive treatments as well as relaxation trainings and most notably psycho-educational group programs have been found effective. Nevertheless, getting appropriate help often turns out to be challenging for cancer patients in routine care.

\section{Take-home message}

Each cancer patient needs appropriate information about his or her disease, including options for somatic and psychological therapy. A screening for mental disorders followed by initiation of adequate therapy results in improved quality of life.

\section{Conflict of interest}

The authors declare that there is no conflict of interest.

\section{References}

[1] Mitchell AJ, Chan M, Bhatti H, et al. Prevalence of depression, anxiety and adjustment disorder in oncological, haematological and palliative-care settings: A meta-analysis of 94 interview-based studies. Lancet Oncol, 12(2): 160-74, 2011.

[2] Fallowfield L, Ratcliffe D, Jenkins V, Saul J. Psychiatric morbidity and its recognition by doctors in patients with cancer. Br J Cancer, 84(8): 1011-5, 2001.

[3] Hjerl K, Anderson EW, Keiding N, et al. Depression as a prognostic factor for breast cancer mortality. Psychosomat, 44(1): 24-30, 2003.

[4] Pelletier G, Verhoef M, Khatri N, Hagen N. Quality of life in brain tumor patients: The relative contributions of depression, fatigue, emotional distress and existential issues. J Neuro-Oncol, 57: 41-49, 2002.

[5] DiMatteo MR, Lepper HS, Croghan TW. Depression is a risk factor for noncompliance with medical treatment. Arch Int Med, 160: 2101-7, 2000.

[6] Prieto JM, Blanch J, Atala J, Carreras E, Rovira M, Cirera E. Psychiatric morbidity and impact on hospital length of stay among hematologic cancer patients receiving stem-cell transplantation. J Clin Oncol, 20: 1907-17, 2002.

[7] Mitchell AJ, Vahabzadeh A, Magruder K. Screening for distress and depression in cancer settings: 10 lessons from 49 years of primarycare research. Psycho-Oncol, 20: 572-84, 2011.
[8] Pollak KI, Arnold RM, Jeffreys AS, et al. Oncologist communication about emotion during visits with patients with advances cancer. J Clin Oncol, 25: 5748-52, 2007.

[9] Hickie IB, Davenport TA, Scott EM, Hadzi-Pavlovic D, Naismith SL, Koschera A. Unmet need for recognition of common mental disorders in Australian general practice. Med J Aust, 175: 18-24, 2001.

[10] Maerckert I, Libert Y, Messin S, Milani M, Slachmuylder J-L, Razavi D. Cancer patients' desire for psychological support: Prevalence and implications for screening patients' psychological needs. PsychoOncol, 19: 141-9, 2009.

[11] Singer S, Bringmann H, Hauss J, et al. Häufigkeit psychischer Begleiterscheinungen und der Wunsch nach psychosozialer Unterstützung bei Tumorpatienten im Aktkrankenhaus. Dtsch Med Wochensch, 132: 2071-6, 2007.

[12] Irwin MR. Depression and risk of cancer progression: An elusive link. J Clin Oncol, 25: 2343-4, 2007.

[13] Jacobi F, Wittchen H-U, Hölting C, et al. Prevalence, co-morbidity and correlates of mental disorders in the general population: Results from the German Health Interview and Examination Survey (GHS). Psychol Med, 34: 1-15, 2004.

[14] Andrykowsky MA, Kangas M. Posttraumatic stress disorder associated with cancer diagnosis and treatment. In: Holland JC, et al., editors. Psycho-Oncology. 2nd ed. Oxford University Press, New York, pp. 348-57, 2010.

[15] Li M, Hales S, Rodin G. Adjustment Disorders. In: Holland JC, et al., editors. Psycho-Oncology. 2nd ed. Oxford University Press, New York, pp. 303-10, 2010

[16] Brown MS, Kroenke MD. Cancer-related fatigue and its associations with depression and anxiety: A systematic review. Psychosomat, 50: 440-7, 2009.

[17] Raison CL, Capuron L, Miller AH. Cytokines sing the blues: Inflammation and the pathogenesis of depression. Trends Immunol, 27(1): 24-31, 2006.

[18] Miller K, Massie MJ. Depressive Disorders. In: Holland JC, et al., editors. Psycho-Oncology. 2nd ed. Oxford University Press, New York, pp. 311-8, 2010.

[19] Massie MJ. Prevalence of Depression in patients with cancer. J Nat Cancer Inst Monogr, 32: 57-71, 2004.

[20] Carlson LE, Bultz BD. Benefits of psychosocial oncology care: Improved quality of life and medical cost offset. Health Qual Life Outcomes, 1(1): 8, 2003.

[21] Holland JC, Bultz BD. National Comprehensive Cancer Network (NCCN). The NCCN guidelines for distress management: A case for making distress the sixth vital sign. J Natl Compr Cancer Netw, 5: 3-7, 2007.

[22] Mitchell AJ. Pooled results from 38 analyses of the accuracy of distress thermometer and other ultra-short methods of detecting cancer-related mood disorders. J Clin Oncol, 25: 4670-81, 2007.

[23] First B, Spitzer RL, Gibbon M, Williams JBW. Structured Clinical Interview for DSM-IV Axis I Disorders, Clinician Version (SCID-CV). American Psychiatric Press, Inc., Washington, D.C. 1996.

[24] Mehnert A, Berg P, Henrich G, Herschbach P. Fear of cancer progression and cancer-related intrusive cognitions in breast cancer survivors. Psychooncol, 18: 1273-80, 2009.

[25] Hjerl K, Andersen EW, Keiding N, Mortensen PB, et al. Increased incidence of affective disorders, anxiety disorders, and non-natural mortality in women after breast cancer diagnosis: A nation-wide cohort study in Denmark. Acta Psychiat Scand, 105(4): 258-64, 2002.

[26] Greenberg DB. Barriers to the treatment of depression in cancer patients. J Natl Cancer Inst Monogr, 32: 127-35, 2004.

[27] Braun IM, William FP. Psychotropic medications in cancer care. In: Holland JC, et al., editors. Psycho-Oncology. 2nd ed. Oxford University Press, New York, 378-85, 2010.

[28] Hellwig S, Merger M, van Calker D. Depressive Störungen bei Tumorpatienten. Pathophysiologie und medikamentöse Behandlungsstrategien. Nervenheilkund, 30: 138-43, 2011.

[29] van Schaik DJF, Klijn AFJ, van Hout HPJ, et al. Patients' preferences in the treatment of depressive disorder in primary care. Gen Hosp Psychiatry, 26 (3): 184-9, 2004.

[30] Temel JS, Greer JA, Muzikansky A, et al. Early palliative care for patients with metastatic non-small-cell lung cancer. N Engl J Med, 363: 733-42, 2010.

[31] Bakitas M, Lyons KD, Hegel MT, et al. Effects of a palliative care intervention on clinical outcomes in patients with advanced cancer. JAMA, 302: 741-9, 2009.

[32] National Health and Medical Research Council (NHMCR). Clinica practice guidelines for the psychosocial care of adults with cancer. NSW, Camperdown: Breast Cancer Center 2003, www.nhmr.gov.au. 